\title{
Role of RGMc as a Neogenin Ligand in Follicular Development in the Ovary
}

\author{
Yu Jin Kim ${ }^{1,+}$, YoungJoon Park ${ }^{2,+}$, Yeo Reum Park ${ }^{1}$, Young Sang Kim ${ }^{1}$, Hye Ran Lee ${ }^{1}$, Sang Jin Lee ${ }^{3}$, \\ Myung Joo Kim ${ }^{1, *}$, KyuBum Kwack ${ }^{2, *(D)}$, Jung Jae Ko ${ }^{2, *}$ and Jae Ho Lee ${ }^{1,2, *(D)}$ \\ 1 CHA Fertility Center Seoul Station, Seoul 04637, Korea; yj_kim@chamc.co.kr (Y.J.K.); \\ summereum@chamc.co.kr (Y.R.P.); p0892@chamc.co.kr (Y.S.K.); hrlee87@chamc.co.kr (H.R.L.) \\ 2 Department of Biomedical Science, CHA University, Seongnam 13488, Gyeonggi-do, Korea; \\ yjparkcb@gmail.com \\ 3 Institute of Animal Genetic Resources Affiliated with Traditional Hanwoo Co., Ltd., Boryeong 33402, Korea; \\ leesj914215@naver.com \\ * Correspondence: splower2@chamc.co.kr (M.J.K.); kbkwack@cha.ac.kr (K.K.); highko@cha.ac.kr (J.J.K.); \\ jaeho@cha.ac.kr (J.H.L.) \\ + These authors contributed equally to this study.
}

Citation: Kim, Y.J.; Park, Y.; Park, Y.R.; Kim, Y.S.; Lee, H.R.; Lee, S.J.; Kim, M.J.; Kwack, K.; Ko, J.J.; Lee, J.H. Role of RGMc as a Neogenin Ligand in Follicular Development in the Ovary. Biomedicines 2021, 9, 280. https://doi.org/10.3390/ biomedicines 9030280

Academic Editor: Giulia Ricci

Received: 23 January 2021

Accepted: 5 March 2021

Published: 10 March 2021

Publisher's Note: MDPI stays neutral with regard to jurisdictional claims in published maps and institutional affiliations.

Copyright: (c) 2021 by the authors. Licensee MDPI, Basel, Switzerland. This article is an open access article distributed under the terms and conditions of the Creative Commons Attribution (CC BY) license (https:/ / creativecommons.org/licenses/by/ $4.0 /)$.
Abstract: There is currently no cure for infertility in women with a poor ovarian response (POR). Neogenin is reported to be abundantly expressed in the ovary; however, its role in mammalian follicular development is unclear and its ligand and signaling pathway remain uncertain. We systematically investigated the role of neogenin and the ligand repulsive guidance molecule $c$ (RGMc) during follicular development. We treated hyperstimulated mouse ovaries with RGMc and analyzed follicular development. Furthermore, we investigated clusters of up/downregulated genes in RGMc-treated ovaries using whole-transcriptome next-generation sequencing (NGS). In addition, we investigated whether expression of up/downregulated factors identified by NGS was also altered in cumulus cells (CCs) of patients with a POR. The number of oocytes was $40 \%$ higher in RGMc-treated ovaries than in control ovaries. NGS data indicated that prostaglandin D2 (PGD2) was involved in the RGMc signaling pathway during follicular development. RGMc treatment significantly elevated the PGD2 level in culture medium of CCs obtained from patients with a POR. Our results demonstrate that RGMc as neogenin ligand promotes follicular development in ovaries via the PGD2 signaling pathway. Therefore, it may be possible to use RGMc for ovarian stimulation in patients with a POR.

Keywords: ovary; neogenin; RGMc; follicular development; PGD2; poor ovarian response

\section{Introduction}

Clinically, a poor ovarian response (POR) is closely related to a low pregnancy success rate and is observed in 9-24\% of infertile female patients [1]. Although great efforts have been made to treat a POR, it cannot be cured or treated with assisted reproductive technologies [2]. The European Society of Human Reproduction and Embryology defined a POR according to the "Bologna Criteria", which are advanced maternal age older than 40 years, a previous low number of collected oocytes with a controlled ovarian stimulation (COS) protocol, and a low anti-Müllerian hormone (AMH) level (0.5-1.1 ng/mL) or an abnormal ovarian reserve test with a follicle count of less than four [3]. There are several POR-specific COS protocols including nature cycle, mild stimulation, and high-dose stimulation [4]. COS is most difficult to perform in patients who do not adequately respond to induction hormones [5]. Therefore, it is critical to improve understanding of primordial follicles and follicle development and to develop specific COS protocols for patients with a POR.

Follicle development is the recruitment of primordial follicles and maturation of antral follicles for ovulation. This process involves multiple cellular complexes between 
oocytes and somatic cells like granulosa cells, cumulus cells [6]. It comprises two phases: the gonadotropin-independent phase and the gonadotropin-dependent phase [6]. The gonadotropin-independent phase is regulated by transforming growth factor $\beta$ family members including bone morphogenetic proteins, growth differentiation factors, activin, inhibin, and $\mathrm{AMH}$ between the primary and secondary follicle stages $[7,8]$. The gonadotropindependent phase is regulated by two gonadotropins, namely, follicle-stimulating hormone (FSH), which stimulates estrogen production, and luteinizing hormone ( $\mathrm{LH})$, which triggers ovulation and development of the corpus luteum during the reproductive cycle [8]. Approaches for overcoming a POR have sought to increase the number of preantral follicles and facilitate the development of antral follicles, which occurs in the gonadotropindependent phase. Induction protocols in patients with a POR target the gonadotropin signaling pathway to increase the number of antral follicles. However, regulatory factors in the gonadotropin-independent and -dependent phases that can be targeted to overcome a POR remain poorly identified.

Neogenin is a member of the immunoglobulin superfamily and functions as a transmembrane receptor with various roles in many tissues such as regulation of neuronal differentiation, inflammation, and tissue regeneration [9-12]. Neogenin has three major signaling ligands: Repulsive Guidance Molecules (RGMs; RGMa, RGMb, and RGMc), netrin-1, and slit [13-15]. These ligands act in various processes such as neurogenesis, mammalian gland morphogenesis, and the apoptosis pathway $[10,13]$. Each ligand signals via a distinct pathway and has different roles depending on the tissue and organ. mRNA expression of neogenin is higher in the ovary than in any other tissue or organ in mammals [16]. Neogenin enhances expression of Oct3/4, Nanog, and Sox2 in preimplantation embryos treated with RGMc $[16,17]$. However, the role of neogenin in follicular development in the ovary is completely unknown [16]. We hypothesized that neogenin controls ovarian physiology.

This study aimed to elucidate the role of neogenin in follicular development by treating hormone (pregnant mare serum gonadotropin (PMSG))-stimulated mouse ovaries with RGMc. We investigated the mechanism by which neogenin and RGMc function in follicular development by performing whole-transcriptome next-generation sequencing (NGS). Finally, we validated the identified neogenin signaling molecules using RGMc-treated cumulus cells (CCs) of patients with a POR to facilitate the development of therapeutic protocols based on the whole transcriptome.

\section{Materials and Methods}

\subsection{Animals}

Outbred white ICR mice (6-8 weeks old, $20-25 \mathrm{~g}$ in weight) were purchased from Oriental Bio (Seongnam, Korea). All procedures for animal breeding and care complied with the regulations of the Institutional Animal Care and Use Committee of CHA University (IACUC No. IACUC200175, 20 October 2020) (Seoul, Korea). In total, 60 mice were used for the experiment $($ control $=30$, test $=30)$.

\subsection{Determination of the Effect of RGMc on Ovaries In Vivo}

For in vivo hormone stimulation, the test group of mice were injected with $0.5 \mathrm{mg} / \mathrm{kg}$ RGMc and then with 75 IU PMSG $12 \mathrm{~h}$ later. The control was only 75IU PMSG injected group. Mice were euthanized by inhalation of $\mathrm{CO}_{2}$ gas in a chamber. Both ovaries were collected, and one was fixed with $4 \%$ paraformaldehyde for histological analysis. The other ovary was stored at $-80^{\circ} \mathrm{C}$ for analysis by whole-transcriptome NGS, RT-PCR, and Western blotting.

\subsection{Histological Analysis and Confocal Imaging}

Mouse ovaries were imaged using light and confocal microscopy. Ovaries were harvested and fixed with $4 \%$ paraformaldehyde for overnight at room temperature. Hematoxylin and eosin (H\&E) staining of ovary was performed using routine protocols. We 
performed H\&E stains with the first cross-section and the 50th cross-section of each sample ovary for counting the number of follicles' development. For immunofluorescence staining, tissues were deparaffinized by incubation with a xylene step, and rehydrated by incubation with a graded ethanol series at room temperature. Slides were costained with a rabbit antineogenin antibody (1:100 diluted, H-175; Santa Cruz Biotechnology, Dallas, TX, USA) and a mouse anti-P63 antibody (1:100 diluted, D-9, Santa Cruz Biotechnology, Dallas, TX, USA). Thereafter, slides were incubated with Alexa Fluor 488- and Alexa Fluor 594-conjugated secondary antibodies (1:200 diluted, Molecular Probes, Eugene, OR, USA). Finally, samples were counterstained with Hoechst 33,342 (H1399; Thermo Fisher Scientific ${ }^{\mathrm{TM}}$, Waltham, MA, USA) for $5 \mathrm{~min}$ at room temperature, washed with PBS, and mounted on cover glasses with anti-fade mounting media (VECTASHIELD H-1000, Burlingame, CA, USA). Confocal microscopic images were acquired using an LSM880 microscope equipped with an Airyscan META device (Carl Zeiss, Oberkochen, Germany).

Oocytes were fixed with $4 \%$ paraformaldehyde for $10 \mathrm{~min}$ at room temperature. The samples were then treated with $0.1 \%$ Triton- $X$ for antigen retrieval and were washed with Phosphate-Buffered Saline (PBS; 14,190,144, pH7.4 Thermo Fisher Life Technologies, San Diego, CA, USA) at room temperature for $30 \mathrm{~min}$. A rabbit anti-neogenin antibody (1:100 diluted in PBS with $0.1 \%$ bovine serum albumin; BSA) was added, followed by incubation overnight at $4{ }^{\circ} \mathrm{C}$. Thereafter, the samples were incubated with the secondary antibody (anti-rabbit labeled 555) for $2 \mathrm{~h}$ at room temperature. The oocytes were washed with PBS. The nuclei were stained with $1 \mu \mathrm{g} / \mathrm{mL} \mathrm{Hoechst}^{\circledR} 33342$ nuclear stain (H1399; Thermo Fisher Life Technologies, San Diego, CA, USA) for $5 \mathrm{~min}$ at room temperature. After washing twice with PBS, the stained oocytes were transferred into a micro-drop of PBS on a glass bottom dish. Images of the stained oocytes were acquired using a confocal microscope (LSM880 with Airyscan META; Carl Zeiss AG, Oberkochen, Germany), and images were analyzed using ZEN2012 software (version 8.0, Carl Zeiss, Oberkochen, Germany).

\subsection{Gene Expression Analysis of RGMc-Treated Ovaries}

Total RNA was isolated from ovaries using TRIzol according to the manufacturer's procedures (15596026; Invitrogen, Waltham, MA, USA). The isolated $100 \mathrm{ng}$ total RNA was transcribed into cDNA using AccuPower ${ }^{\circledR}$ CycleScript RT PreMix (Bioneer, Daejeon, Korea) and then amplified with AccuPower ${ }^{\circledR}$ Taq PCR PreMix (Bioneer) using primer sets specific for mouse neogenin, Oct3/4, Nanog, p63, and $\beta$-actin (Table S1). In total, $10 \mathrm{pmol} / \mu \mathrm{L}$ forward and reverse primers and $200 \mathrm{ng}$ template cDNA were added to AccuPower ${ }^{\circledR}$ Taq PCR PreMix tubes, and then distilled water was added up to a total volume of $20 \mu \mathrm{L}$. The PCR cycling conditions were as follows: 1 min at $95^{\circ} \mathrm{C}$, followed by 32 cycles of denaturation for $30 \mathrm{~s}$ at $95^{\circ} \mathrm{C}$, annealing for $30 \mathrm{~s}$ at $60^{\circ} \mathrm{C}$, and extension for $50 \mathrm{~s}$ at $72{ }^{\circ} \mathrm{C}$. PCR products were resolved on $2 \%$ agarose gels with Safeview ${ }^{\mathrm{TM}}$ (Applied Biological Materials, Richmond, BC, Canada). The gels were visualized under ultraviolet illumination using a gel documentation system (WSE-6100 LuminoGraph; ATTO, Tokyo, Japan).

\subsection{Protein Expression Analysis of RGMc-Treated Ovaries}

Protein expression in RGMc-treated ovaries was analyzed by Western blotting. Ovarian tissue samples were homogenized in PRO-PREPTM Protein Extraction Solution (iNtRON Biotechnology, Seoul, Korea). Proteins were denatured at $95{ }^{\circ} \mathrm{C}$ for $5 \mathrm{~min}$ in Laemmli buffer, separated by $10 \%$ sodium dodecyl sulfate polyacrylamide gel electrophoresis, and transferred onto $0.2 \mu \mathrm{m}$ nitrocellulose membranes (Bio-Rad, Hercules, CA, USA). The membranes were blocked in TBST buffer containing 5\% BSA for $1 \mathrm{~h}$ at room temperature and then incubated with rabbit anti-neogenin polyclonal (NBP1-89651, 1:2000; Novus, Centennial, CO, USA), rabbit anti-Oct3/4 (sc-9081, 1:2000; Santa Cruz Biotechnology, Dallas, TX, US), mouse anti-Nanog (sc-134218, 1:2000; Santa Cruz Biotechnology), mouse anti-p63 (sc-25268, 1:2000; Santa Cruz Biotechnology, Dallas, TX, USA), and mouse anti- $\beta$-actin (MA5-15739, 1:2000; Invitrogen, Waltham, MA, US) primary antibodies diluted in TBST buffer containing $1 \%$ BSA overnight at $4{ }^{\circ} \mathrm{C}$. After washing with TBST buffer, the mem- 
branes were incubated with horseradish peroxidase-conjugated donkey anti-mouse and anti-rabbit IgG secondary antibodies (5178-2504 and 5196-2504; Bio-Rad). Immunoreactive bands were visualized using enhanced chemiluminescence (Clarity ${ }^{\mathrm{TM}}$ Western ECL Substrate 1,705,060, Bio-Rad). Images of the bands were acquired using a gel documentation system (WSE-6100 LuminoGraph I, ATTO).

\subsection{Whole-Transcriptome NGS Analysis Comparing Control and RGMc-Treated Ovaries}

The whole transcriptomes of RGMc-treated and control ovaries were analyzed by NGS. Total RNA was isolated from ovaries using TRIzol following the manufacturer's protocols (15,596,026; Invitrogen, Waltham, MA, USA). Purified mRNA was used for NGS after its quality had been checked. Sample sheets were prepared on the MiSeq sequencer (Illumina, San Diego, CA, USA) to provide run details. NGS data were analyzed using the REVIGO program and Gene Ontology.

Differentially expressed gene (DEG) and enrichment analyses of RGMc-treated and control ovaries were performed using four Mus musculus samples per group. The raw sequencing data were mapped, and potential transcripts were assembled using HISAT2 (ver. 2.1.0), Bowtie2 (ver. 2.3.4.1), and StringTie (ver. 1.3.4 d). The aligned read counts were normalized with the Trimmed Mean of M-values method in edgeR. Genes whose expression exhibited an absolute fold-change of 4 or higher were extracted and clustered by an agglomerative method with Euclidean and Ward methods.

A biological process database comprising 20,196 reference genes was used for gene enrichment analyses. The ClueGO (ver. 2.5.5) module of Cytoscape (ver. 3.7.2) was used to analyze pathway enrichment for each up/downregulated gene in RGMc-treated ovaries. Significance in enrichment analysis was determined using a two-sided hypergeometric test with Bonferroni step-down corrections. A term with an enrichment percentage higher than $60 \%$ was selected for each up/downregulated gene cluster. The kappa score was calculated to identify associations between enriched terms.

\subsection{RT-qPCR to Verify Gene Expression Changes in Ovaries}

RT-qPCR was performed to confirm the NGS data obtained for control and RGMctreated ovaries. Based on the KEGG pathway, primers targeting upregulated (Ptgs1, Edn2, and Hpgds) and downregulated (Tbxa2r, Oxtr, and Adra1d) genes were selected (Table S1). RT-qPCR was performed with total RNA isolated from ovaries using TRIzol. Total RNA was reverse-transcribed into cDNA using AccuPower ${ }^{\circledR}$ CycleScript RT PreMix (Bioneer). qPCR was performed using SsoAdvanced Universal SYBR Green Supermix (\#1725270, Bio-Rad) on a spectrofluorometric thermal cycler (CFX96 Touch Real-Time PCR Detection System, Bio-Rad). The reaction contained $10 \mathrm{pmol} / \mu \mathrm{L}$ forward and reverse primers, $200 \mathrm{ng}$ template cDNA, and $2 \times$ SsoAdvanced Universal SYBR Green Supermix. Distilled water was added up to a final volume of $20 \mu \mathrm{L}$. The PCR cycling conditions were as follows: $3 \mathrm{~min}$ at $95^{\circ} \mathrm{C}$, followed by 40 cycles of denaturation for $10 \mathrm{~s}$ at $95^{\circ} \mathrm{C}$, annealing for $30 \mathrm{~s}$ at $60{ }^{\circ} \mathrm{C}$, and extension for $20 \mathrm{~s}$ at $72{ }^{\circ} \mathrm{C}$. Expression of each examined gene was normalized to $\beta$-actin. Triplicate samples were tested.

\subsection{Validation Study Using Human CCs and Follicular Fluid}

NGS data indicated that prostaglandins were key elements in the effects of RGMc on follicular development. Therefore, the relationship of prostaglandins with a POR was investigated using human follicular fluid and CCs (10 samples). Follicular fluid was collected during ovum pick-up and CCs were collected after denuding of oocytes. The collection of human material was approved by the Institutional Research and Ethical Committees of CHA University (approval number: 1044308-201611-BR-027-04, 29 June 2020). An enzymelinked immunosorbent assay (ELISA) was performed using follicular fluid collected from patients with a normal ovarian response and patients with a POR, which was characterized by pick-up of fewer than four oocytes and an AMH level $\leq 1 \mathrm{ng} / \mathrm{mL}$. The concentration of prostaglandin D2 (PGD2) (sensitivity: $9.38 \mathrm{pg} / \mathrm{mL}$, no significant cross-reactivity or 
interference between PGD2 and analogues was observed. E4718; Biovision, Milpitas, CA, USA) in follicular fluid was determined with an ELISA kit (Abcam, Cambridge, UK) following the manufacturer's protocol. Signals were read with a microplate reader (Epoch microplate spectrophotometer, BioTek Instruments, Inc., Winooski, VT, USA). Gene expression in CCs was analyzed by real-time PCR. Each sample mRNA was isolated from CCs of patients with a normal ovarian response and patients with a POR using a DynaBeads mRNA DIRECT Kit (Life Technologies, Oslo, Norway). mRNA was quantified using a NanoDrop ND-1000 spectrophotometer (Nyxor Biotech, Paris, France). Total RNA was reverse-transcribed into cDNA using AccuPower ${ }^{\circledR}$ CycleScript RT PreMix (Bioneer) with poly-dT. In total, $10 \mathrm{pmol} / \mu \mathrm{L}$ forward and reverse primers and $200 \mathrm{ng}$ template cDNA were added to AccuPower ${ }^{\circledR}$ Taq PCR PreMix tubes and then distilled water was added up to a total volume of $20 \mu \mathrm{L}$. The human primer sets are mentioned in Table S2. The PCR cycling conditions were as follows: $1 \mathrm{~min}$ at $95^{\circ} \mathrm{C}$, followed by 32 cycles of denaturation for $30 \mathrm{~s}$ at $95^{\circ} \mathrm{C}$, annealing for $30 \mathrm{~s}$ at $58^{\circ} \mathrm{C}$, and extension for $50 \mathrm{~s}$ at $72^{\circ} \mathrm{C}$. PCR products were resolved on $2 \%$ agarose gels with Safeview ${ }^{\mathrm{TM}}$ (Applied Biological Materials). The gels were visualized under ultraviolet illumination using a gel documentation system (WSE-6100 LuminoGraph; ATTO).

\subsection{RGMc Treatment of CCS Obtained from Patients with a POR}

To investigate the effect of RGMc on CCs, samples were collected from eight control patients and eight patients with a POR. The latter had been diagnosed with infertility due to a POR according to the Bologna criteria, while the former had a normal ovarian response and normal ovarian function. All patients underwent conventional COS protocols with a gonadotropin-releasing hormone antagonist during in vitro fertilization (IVF) procedures. CCs were seeded into a 96-well plate at a density of $10^{4}$ cells/well in QCL media. The next day, the culture medium was replaced with that containing $50 \mathrm{ng} / \mathrm{mL}$ RGMc. After 1 day, the culture medium was harvested and measured the concentration of PGD2 in the media using ELISA kits.

\subsection{Statistical Analysis}

All data are expressed as the means \pm standard error of the mean (SEM) of triplicate measurements. Statistical analyses were carried out using a one-way ANOVA analysis with Bonferroni test of variance. The significance level was set at ${ }^{*} p<0.05,{ }^{* *} p<0.01$, and *** $p<0.001$. Significant differences are indicated by asterisks in each figure.

\section{Results}

\subsection{Neogenin Expression in Mouse Ovaries and Oocytes}

Expression of neogenin in mouse ovaries and oocytes was examined by immunofluorescence staining. Neogenin was expressed at all stages of follicular development (Figure 1a). Staining with an anti-neogenin antibody (red) was detected in immature oocytes at the germinal vesicle and metaphase II stages (Figure 1b). Neogenin was expressed at the mRNA (Figure 1c) and protein (Figure 1d) levels in mouse ovaries.

\subsection{RGMc Treatment Enhances Follicular Development in Hormone-Stimulated Mouse Ovaries}

We evaluated the effect of RGMc treatment on mouse ovaries stimulated with PMSG in vivo. Figure 2a shows histological images of mouse ovarian tissues stained with hematoxylin and eosin following RGMc treatment and hormone stimulation. The number of total follicles, including preantral and antral follicles, was significantly higher in RGMctreated ovaries than in control ovaries (Figure 2b). The number of antral follicles was 2.5-fold higher in RGMc-treated ovaries than in control ovaries (Figure 2c). Consequently, twice as many oocytes were collected from RGMc-treated ovaries than from control ovaries (Figure 2d). In summary, treatment with the neogenin ligand RGMc enhanced follicular development in the ovary. 
a



Secondary follicle

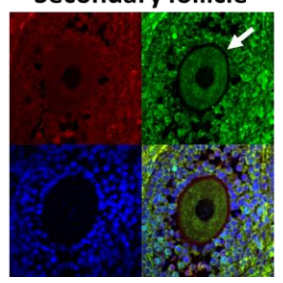

Primary follicle

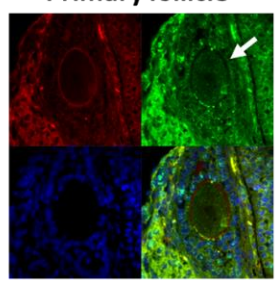

Oocyte in G. follicle

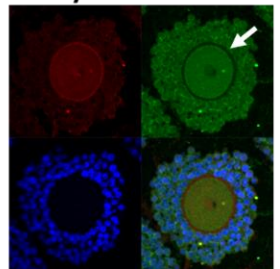

b

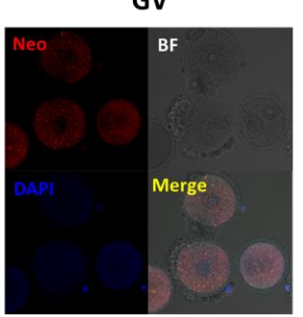

c

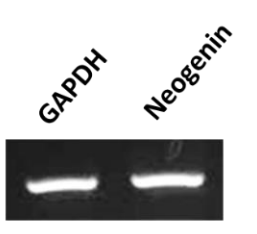

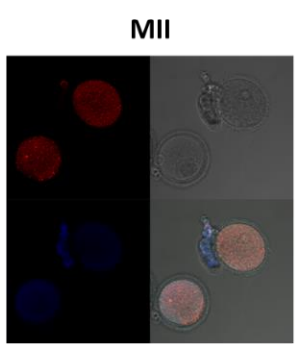

d

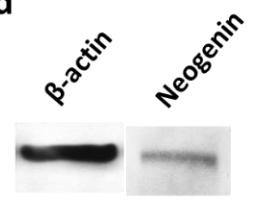

Figure 1. Expression profiling of neogenin in the ovary. (a) Confocal microscopic images of immunofluorescence staining with an anti-neogenin antibody in primordial follicle, primary follicle, secondary follicle, and oocyte in graafian follicle (oocyte in G. follicle). Neogenin, p63, and DAPI staining is shown in green, red, and blue, respectively. The arrow indicates a follicle positively stained for neogenin. (b) Confocal microscopic images of mouse oocytes. Neogenin and DAPI staining is shown in red and blue, respectively. GV, germinal vesicle; MII, metaphase II; BF, bright field. (c) RT-PCR analysis of neogenin mRNA in mouse oocytes. GAPDH was used as an internal control. (d) Western blot analysis of neogenin protein in mouse ovaries. $\beta$-actin was used as an internal control. Neo: Neogenin. Scale bar $=100 \mu \mathrm{m}$. All data are representative of three replicates.

a

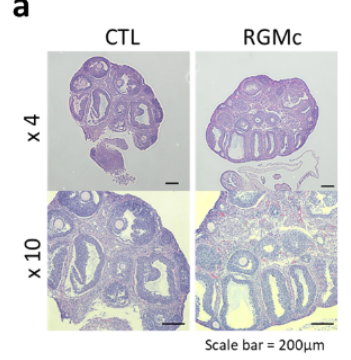

b

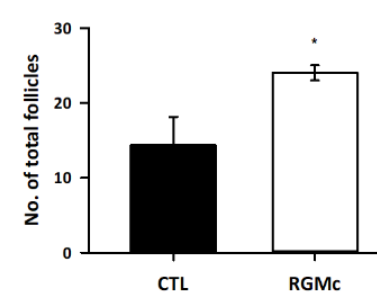

C

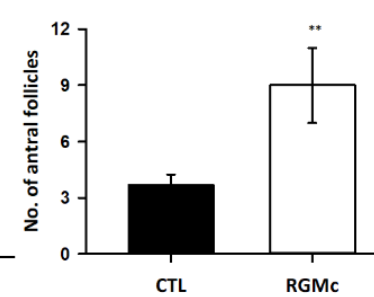

d



Figure 2. Characterization of RGMc-treated ovaries. (a) Light microscopic images of hematoxylin and eosin-stained mouse ovarian tissue treated without (CTL) and with RGMc. Scale bar $=200 \mu \mathrm{m}$. (b-d) Graphs of the total number of follicles (b), number of antral follicles (c), and number of collected oocytes (d) in control (CTL) and RGMc-treated ovaries. Data are the mean \pm SEM of three replicates. ${ }^{*} p<0.05$ and ${ }^{* *} p<0.005$ versus the control group.

3.3. Expression of the Primordial Follicle Factors Oct3/4, Nanog, p63, and Neogenin Is Increased in RGMc-Treated Mouse Ovaries

We analyzed expression of the primordial follicle factors Oct3/4, Nanog, p63, and neogenin in RGMc-treated mouse ovaries by RT-PCR. mRNA expression of Oct3/4, Nanog, p63, and neogenin was significantly higher in RGMc-treated ovaries than in control ovaries (Figure 3a). In particular, mRNA expression of Oct3/4, Nanog, and p63 was 2-fold higher in RGMc-treated ovaries than in control ovaries (Figure 3b). Protein expression of Oct3/4, Nanog, p63, and neogenin was significantly higher in RGMc-treated ovaries than in control ovaries (Figure 3c).

\subsection{Whole-Transcriptome and Gene Enrichment Analyses of the Effects of RGMc Treatment on Hormone-Stimulated Mouse Ovaries}

Transcriptomic differences between RGMc-treated and control ovaries were investigated by RNA-sequencing. mRNA expression of 275 genes differed by more than 4 -fold between RGMc-treated and control ovaries. Among these DEGs, 197 and 78 DEGs were upregulated in RGMc-treated and control ovaries, respectively (Figure 4a,b). 
a

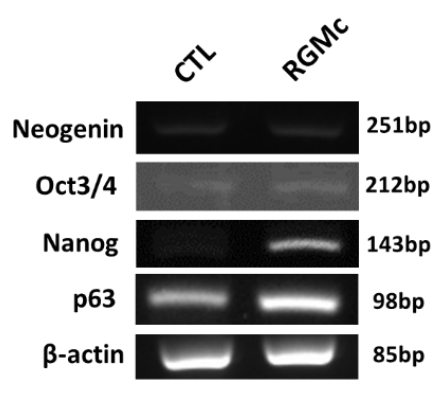

b

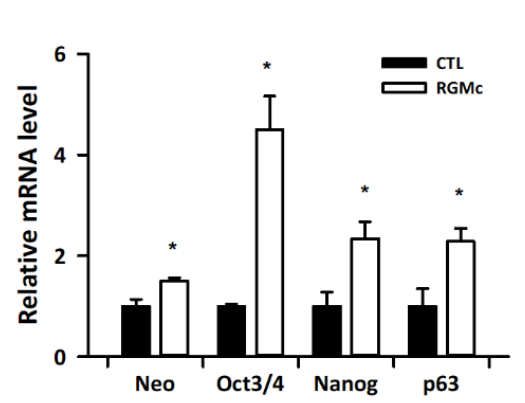

c

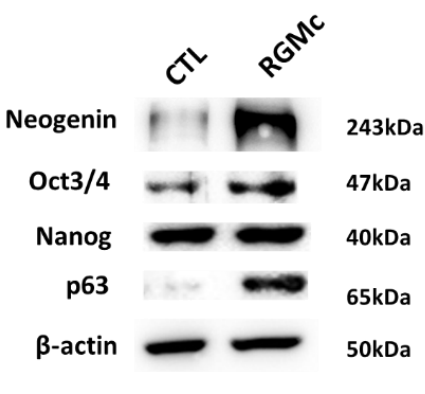

Figure 3. mRNA and protein expression in RGMc-treated ovaries. (a) RT-PCR analysis of neogenin (Neo), Oct3/4, Nanog, and p63 expression in control (CTL) and RGMc-treated ovaries. (b) Quantitative real-time PCR analysis of relative expression of neogenin (Neo), Oct3/4, Nanog, and p63 in control (CTL) and RGMc-treated ovaries. (c) Western blot analysis of neogenin (Neo), Oct3/4, Nanog, and p63 expression in control (CTL) and RGMc-treated ovaries. $\beta$-actin was used as an internal control. Data are the mean \pm SEM of three replicates. ${ }^{*} p<0.05$ versus the control group.
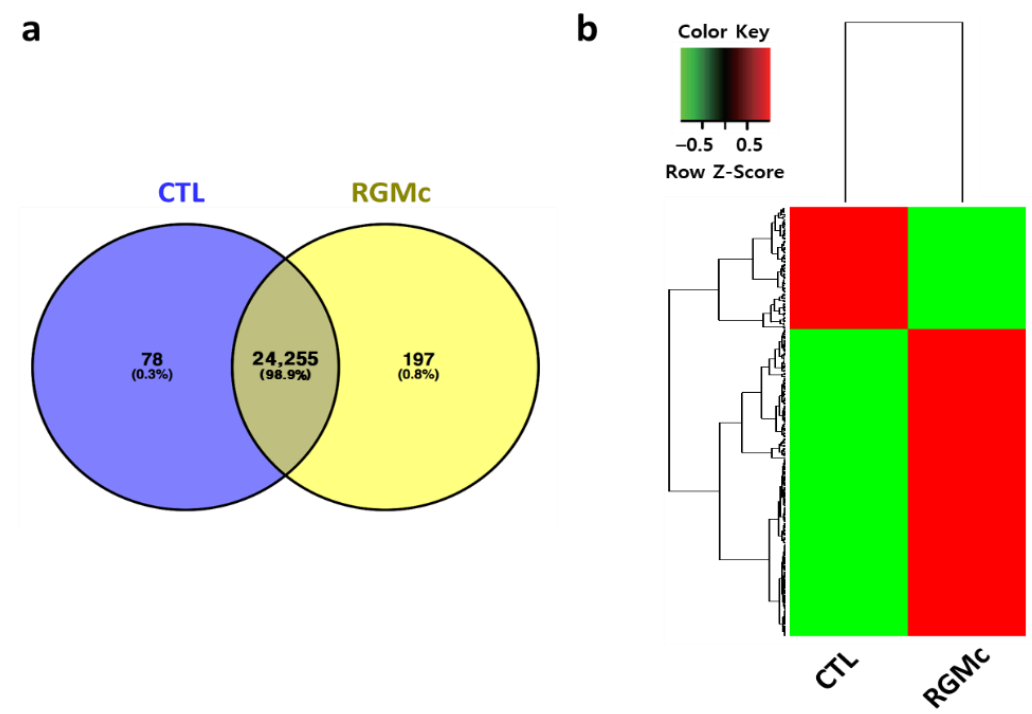

Figure 4. Whole-transcriptome NGS of RGMc-treated ovaries. (a) Venn diagram of up/downregulated genes (fold-change $>4$ ) in control and RGMc-treated ovaries. A total of 24,255 genes were included in RNA-sequencing analysis. (b) Cluster map of up/downregulated genes (fold-change $>4$ ).

To elucidate the mechanisms underlying hyperovulation in RGMc-treated ovaries, gene enrichment analysis of these 197 and 78 DEGs was performed. Enriched terms were determined using the ClueGO module of Cytoscape. To identify the significance of enrichment, a hypergeometric test with Bonferroni step-down corrections was applied, and 23 terms were selected. Among the 275 DEGs, 54 DEGs, including 35 upregulated and 19 downregulated DEGs, were annotated to the 23 terms (Figure 5a, Figure S1, Table S3). Protein-protein interactions for these terms were determined by Gene Ontology analysis. To determine the key genes, up/downregulated DEGs associated with the terms were plotted together using the ClueGO and CluePedia modules of Cytoscape (Figure 5b). Thicker lines represent relationships based on experimental evidence. Thinner lines represent relationships based on all other types of evidence. The size and color of circles represent the significance of enrichment and the percentage of up/downregulated genes, respectively. Among the 35 upregulated DEGs, 23 were annotated to eight cilium- and microtubulerelated terms (cilium organization, cilium organization, cilium assembly, microtubule-based movement, microtubule bundle formation, axoneme assembly, axonemal dynein complex assembly, and inner dynein assembly) and were closely interconnected (Figure 5b left). The remaining 12 upregulated DEGs and all 19 downregulated DEGs were annotated to seven downregulated, six upregulated, and two nonsatisfied terms (Figure 5b right). 


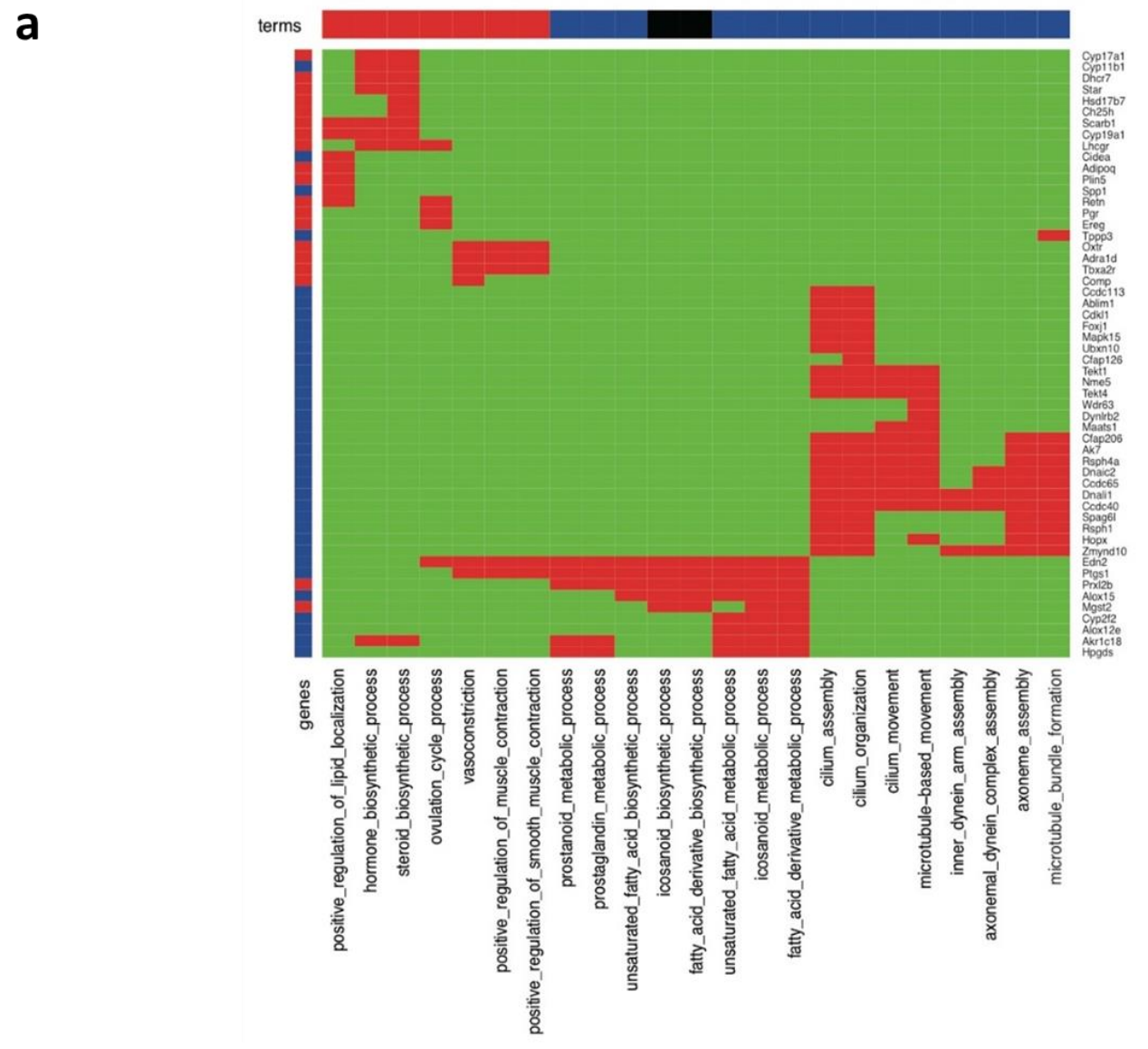

b

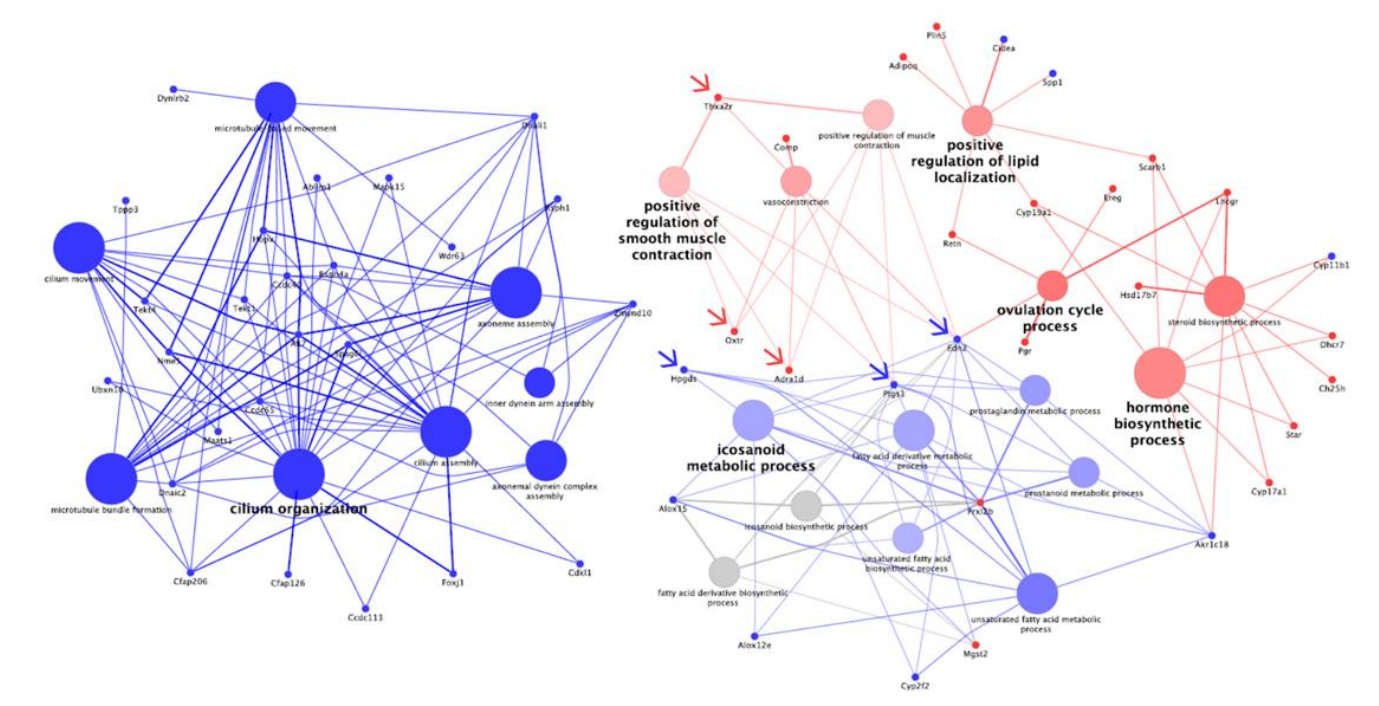

Figure 5. Up/downregulated genes based on an absolute fold-change of more than 4 in RGMc-treated ovaries. (a) Heatmap based on a binary code for significantly enriched terms and up/downregulated genes in RGMc-treated ovaries. Blue and red in the top and side bars represent upregulated and downregulated terms in RGMc-treated ovaries, respectively. Black in the top and side bars represents nonsatisfied terms with higher than $60 \%$ enrichment for up/downregulated genes. Red and green in the heatmap represent the presence and absence of the gene, respectively. (b) Network between significantly enriched terms and up/downregulated genes (fold-change $>4$ ). Blue and red represent upregulated and downregulated genes/terms in RGMc-treated ovaries, respectively. Gray represents nonsatisfied terms with higher than $60 \%$ enrichment for up/downregulated genes. Blue and red arrows indicate target genes for validation that were upregulated and downregulated upon RGMc treatment, respectively. 
3.5. Experimental Validation of Changes in Expression of Prostaglandin and Muscle Contraction-Related Genes upon RGMc Treatment in Mice

After performing transcriptomic analyses, we experimentally validated the changes in mRNA expression of three upregulated (Figure 5b, blue arrows) and three downregulated (Figure 5b, red arrows) genes upon RGMc treatment by real-time qPCR (Figure 6a). Among these genes, mRNA expression of hematopoietic prostaglandin D synthase (Hpgds) and endothelin 2 ( $E d n 2$ ) was more than 13-fold significantly higher in RGMc-treated ovaries than in control ovaries $(p<0.05)$, while mRNA expression of a downregulated gene, namely, thromboxane A2 receptor (Tbxa2r), was significantly decreased in RGMc-treated ovaries $(p<0.05)$ (Figure 6b). In addition, although not statistically significant, oxytocin receptor (Oxtr) was also markedly downregulated in RGMc-treated ovaries (Figure 6a,b).

a

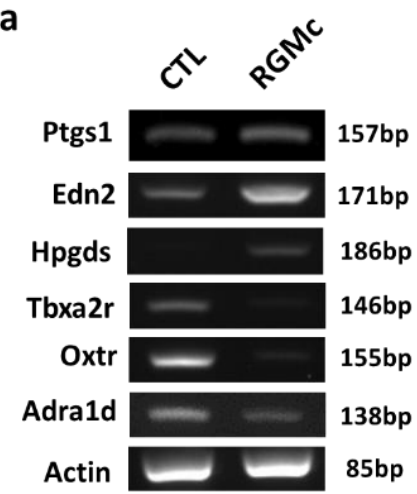

b

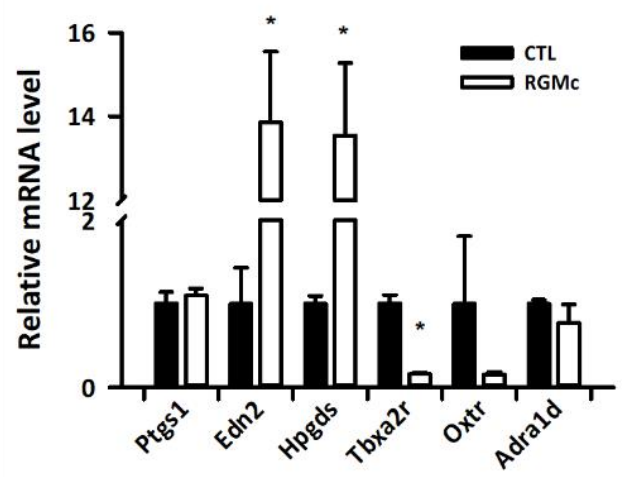

Figure 6. Quantitative real-time PCR analyses to validate the NGS data in RGMc-treated ovaries. (a) RT-PCR analysis of Ptgs1, Edn2, Hpgds, Tbxa2r, Oxtr, Adra1d, and $\beta$-actin expression in control (CTL) and RGMc-treated ovaries. (b) Quantitative real-time PCR analysis of Ptgs1, Edn2, Hpgds, Tbxa2r, $O x t r$, and Adra1d expression in control (CTL) and RGMc-treated ovaries normalized against $\beta$-actin expression. ${ }^{*} p<0.05$ versus the control group.

3.6. The Level of PGD2 in Follicular Fluid and Culture Medium of RGMc-Treated CCs Obtained from Patients with a POR

We analyzed the level of PGD2 in the follicular fluid of patients with a normal ovarian response and patients with a POR using an ELISA. The PGD2 concentration was significantly lower in the follicular fluid of patients with a POR than in the follicular fluid of young and old patients with a normal ovarian response (Figure 7a). Next, we confirmed that CCs of normal and POR patients expressed neogenin and therefore analyzed the effect of RGMc treatment on CCs obtained from patients with a normal ovarian response and patients with a POR (Figure 7d). Treatment with RGMc significantly increased the PGD2 level in the culture medium of CCs obtained from patients with a POR by 4-fold (Figure 7c) and also dramatically increased the PGD2 level in the culture medium of CCs obtained from patients with a normal ovarian response (Figure $7 \mathrm{~b}$ ). 
a

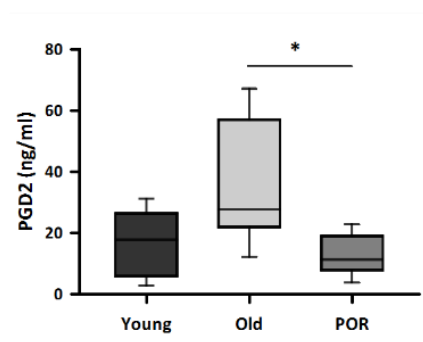

b

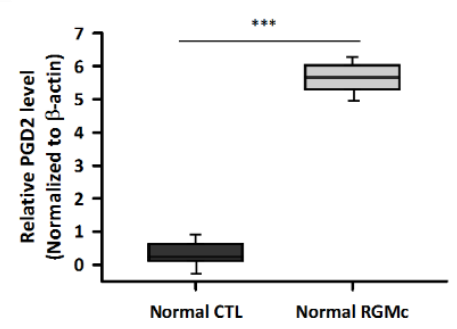

C

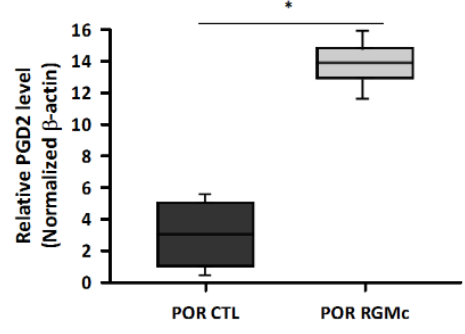

d

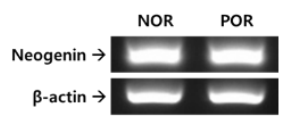

Figure 7. An ELISA of PGD2 in human follicular fluid and the culture medium of RGMc-treated CCs. (a) The PGD2 level in follicular fluid of patients with a POR and young and old patients with a normal ovarian response. (b,c) The ELISA data of PGD2 level in the culture media of RGMc-treated CCs obtained from (b) patients with a normal ovarian response and (c) patients with a POR. (d) Gene expression of neogenin in human CCs of normal and POR patients. All assays were repeated three times with different samples for statistical analysis. ${ }^{*} p<0.05$ and ${ }^{* * *} p<0.001$ versus the young and old normal ovarian response groups.

\section{Discussion}

In this study, we found that neogenin and the ligand RGMc are involved in both the gonadotropin-independent and -dependent phases of follicular development. Neogenin is expressed at various stages of follicular development in the ovary from primordial follicles to graafian follicles. Thus, RGMc treatment enhanced both the gonadotrophin-independent and -dependent phases upon hormone hyperstimulation. Our results demonstrated that RGMc treatment enhanced the activity of primordial follicles. Oct3/4, Nanog, and p63 are markers of primordial follicles and regulate the proliferation of stromal cells and their differentiation of granulosa cells supplement into preantral follicles development [18] RGMc may facilitate COS by upregulating Oct $3 / 4$ and Nanog, thereby enhancing survival of primordial follicles in the ovary. p63 is a regulator of meiosis and is important for cell cycle control in primordial follicles [19]. RGMc treatment promoted follicular development in the ovary. In particular, it upregulated prostaglandins including PGD2. Prostaglandins regulate both the gonadotrophin-independent and -dependent phases in the ovary. In summary, RGMc promoted follicular development by binding to neogenin and upregulating PGD2.

We investigated the mechanism by which RGMc treatment enhances follicular development in the ovary by performing whole-transcriptome NGS. This revealed that 197 and 78 genes were upregulated and downregulated in RGMc-treated ovaries, respectively. We determined which terms were significantly enriched among the upregulated genes (fold-change $>4$ ). RGMc-treated ovaries exhibited upregulation of genes involved in microtubule formation and cilia organization [20-22]. Microtubules in granulosa cells have been linked with cholesterol movement for steroidogenesis in mitochondria upon FSH stimulation [22]. Cilia function in steroidogenesis to produce various molecules, such as estrogen in granulosa cells via Ift88 [20]. Mice with conditional knockdown of Ift 88 exhibit abnormal estrogen production in the ovaries and defects in ovarian function. A series of adenylate kinases including AK7 is translated in germinal vesicle-stage oocytes and linked with cilia function for steroidogenesis [23]. RGMc treatment upregulated $E d n 2$ and genes involved in microtubule bundle formation. There are three endothelin proteins called EDN1, EDN2, and EDN3. These vasoactive peptides contain 21 amino acids and reportedly function in various processes in the ovary, including steroidogenesis, follicles development, and ovulation [24]. During the reproductive cycle, EDN2 functions in the rupture of the follicle wall by regulating blood pressure and corpus luteum formation in the ovary $[25,26]$. Edn2-knockout mice exhibit a poor ovulatory response to superovulation induction [26]. 
Our data demonstrate that RGMc treatment enhanced follicular development by upregulating $E d n 2$. The role of $E d n 2$ in ovulatory competence requires further investigation. RGMc treatment also upregulated Hpgds in the ovary. HPGDS produces PGD1 and PGD2, thereby interfering with the actions of FSH in granulosa cells [27]. Signaling of PGD2 produced by HPGDS controls expression of the FSH and LH receptors [27]. PGD2 modulate the balance between proliferation, differentiation, and steroidogenic activity in granulosa cells during the gonadotropin-independent and -dependent phases, and are thus important for the ovulatory cascade including oocyte maturation, cumulus expansion, and follicle maturation [28].

Downregulation of genes in RGMc-treated ovaries prevents premature ovulation and facilitates normal follicles development. One downregulated gene cluster was Oxtr signaling, which is associated with various biological functions in female reproductive organs. Oxytocin and Oxtr, which function in steroidogenesis, are associated with a POR, and their levels are elevated in the follicular fluid of women with PCOS [29-31]. Endogenous oxytocin might be involved in the regulation of LH in women [32]. However, the role of Oxtr is unclear, and further studies are required to elucidate its relationship with follicles development. Tbxa2r was also downregulated in RGMc-treated ovaries. However, $T b x a 2 r$ has not been investigated in the ovary, and a further study is required to determine the significance of its downregulation in reproductive organs.

In the validation study, we measured the concentrations of prostaglandins in human follicular fluid and found that PGD2 supports ovarian function and female fertility. PGD2 is produced by HPGDS in granulosa cells. It plays an important role and modulates the proliferation and differentiation of granulosa cells and steroidogenic activity in these cells. Our data demonstrated that the PGD2 level was lower in the follicular fluid of patients with a POR than in the follicular fluid of patients with a normal ovarian response. A POR was associated with prostaglandin metabolic defects. RGMc treatment may be able to overcome a POR for COS during IVF cycles. The PGD2 signaling pathway may play a key functional role in normal follicular development upon hormone stimulation during IVF cycles. We suggest that PGD2 is a potential biomarker to diagnose a POR. Treatment with RGMc dramatically increased the PGD2 level in the culture medium of CCs obtained from patients with a POR. These data show a possibility for PORs therapeutic factor which is promoted follicular development and that RGMc can be used to develop new COS protocols for personalized induction in women with a POR.

Taken together, RGMc affects the gonadotropin-independent and -dependent phases of follicular development via neogenin signaling. We found that neogenin in the ovary is associated with several signaling molecules including EDN2, HPGDS, and PGD2 (Figure 8). Moreover, RGMc treatment significantly increased the level of PGD2 in the culture medium of human CCs. CCs are important for follicles development and oocyte quality as supporting cells in the ovary [33-35], which are responsible for nurturing oocyte growth, development, and the gradual acquisition of developmental competence [36]. Therefore, the metabolic activity of CCs upon RGMc treatment may improve oocyte competence to generate good-quality preimplantation embryos.

In conclusion, neogenin has a potential role in primordial follicles and follicular development in the ovary. Despite many studies, an efficient stimulation protocol for patients with a POR is lacking. Most treatments that improve pregnancy rates have not been recommended in patients with a POR, and a POR remains one of the most challenging conditions to treat in reproductive medicine. New COS protocols incorporating RGMc should be investigated to enhance follicles development for IVF in infertile female patients with a POR. 


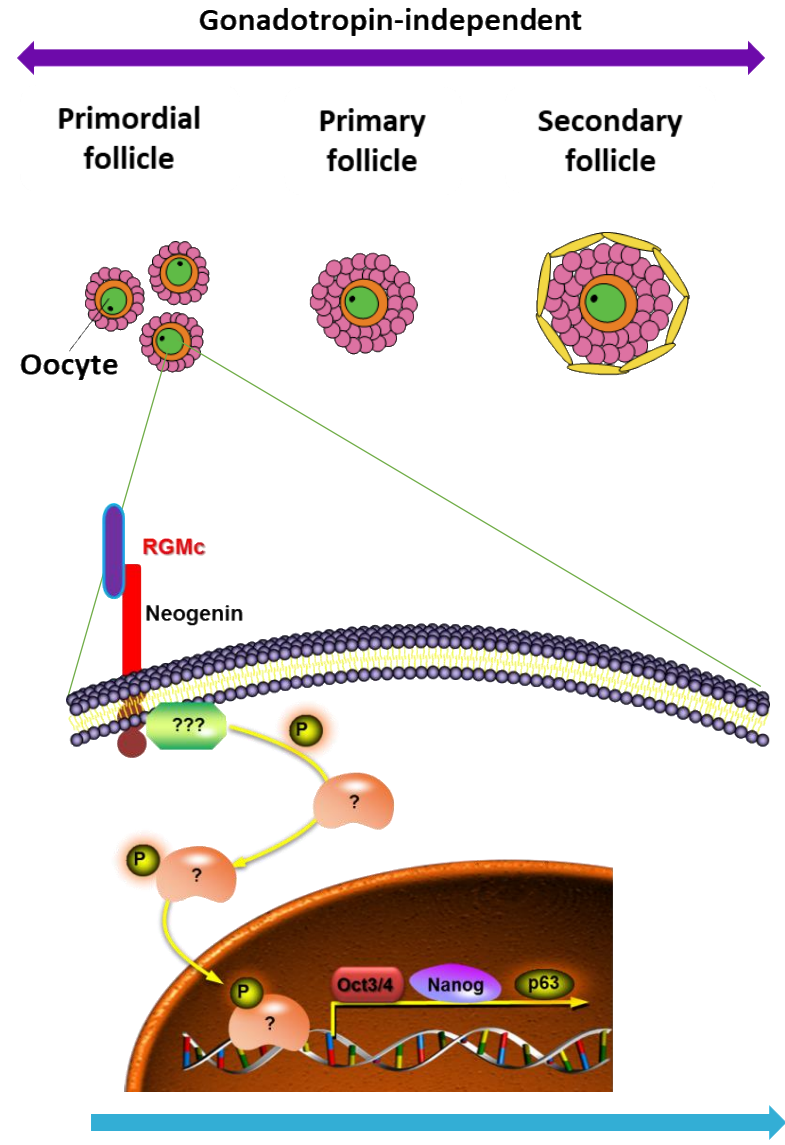

UP : Oct3/4, Nanog, p63

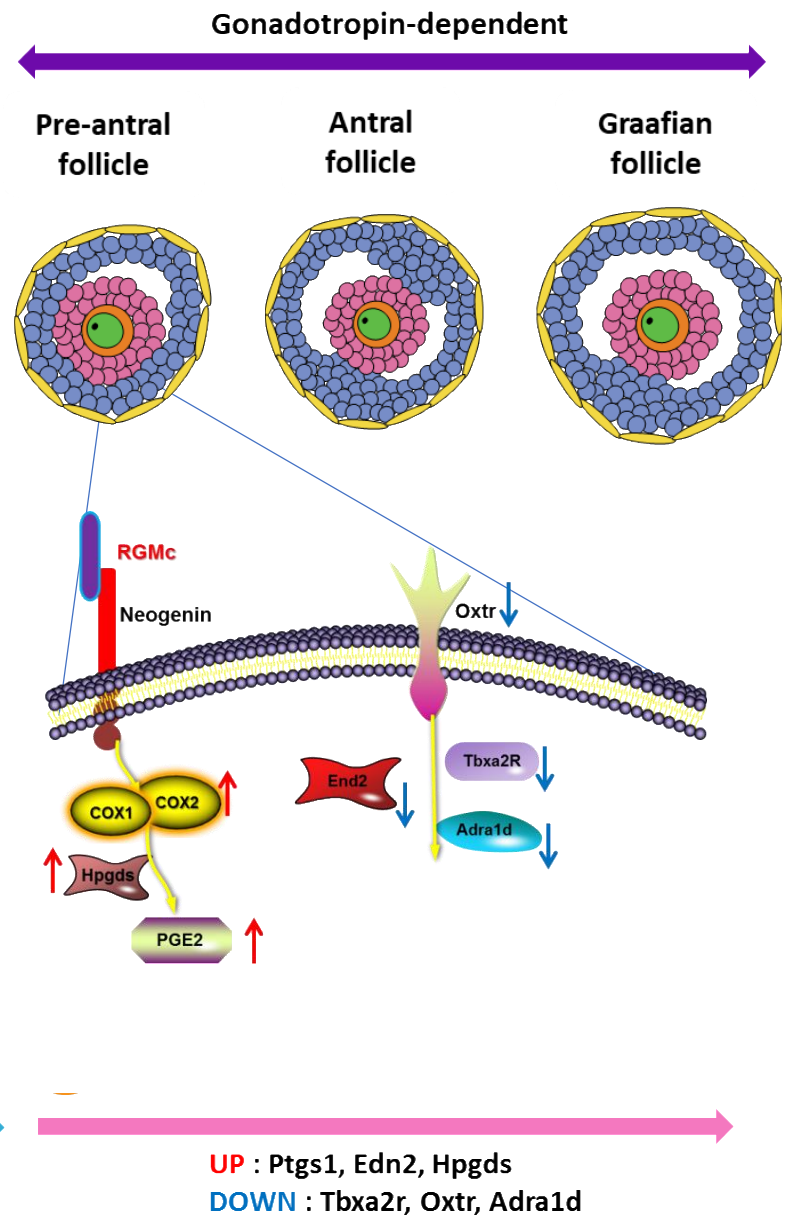

Figure 8. Schematic illustration of the signaling pathway by which the neogenin ligand RGMc enhances follicular development.

Supplementary Materials: The following are available online at https:/ / www.mdpi.com/2227-905 9/9/3/280/s1. Figure S1: Pairwise cluster map of kappa scores with each significant term. Table S1: Primer sequences used for real-time qPCR of RGMc-treated mouse ovaries. Table S2: Primer sequences used for qPCR of RGMc-treated human CCs. Table S3: Protein-protein interactions (PPIs) for the gene enrichment terms of DEGs determined using Gene Ontology. Of 197 upregulated (yellow and orange) and 78 downregulated (blue and green) DEGs, 35 and 19 DEGs were associated with the terms, respectively.

Author Contributions: Y.J.K., Y.P. and Y.R.P. contributed to acquisition, analysis, and interpretation of data; Y.S.K., H.R.L. and M.J.K. contributed to sample collection and design; S.J.L. contributed to experimental design; K.K., J.J.K. and J.H.L. contributed to study conception and design, and writing of the manuscript. All authors have read and agreed to the published version of the manuscript.

Funding: This study was supported by the Basic Science Research Program through the National Research Foundation of Korea (NRF) [grant numbers 2018R1D1A1B07050138, 2018R1D1A1B07044016, and 2019R1A2C1086882].

Institutional Review Board Statement: The study was conducted according to the guidelines of the Declaration of Helsinki, and approved by the Institutional Animal Care and Use Committee of CHA University (IACUC No. IACUC200175, 20 October 2020).

Informed Consent Statement: Informed consent was obtained from all subjects involved in the study.

Conflicts of Interest: The authors declare no conflict of interest. 


\section{References}

1. Gorgy, A.; Naumann, N.; Bates, S.; Craft, I.L. Assisted conception following poor ovarian response to gonadotrophin stimulation. Br. J. Obstet. Gynaecol. 1997, 104, 1420-1421. [CrossRef] [PubMed]

2. Papathanasiou, A.; Searle, B.J.; King, N.M.; Bhattacharya, S. Trends in poor responder research: Lessons learned from RCTs in assisted conception. Hum. Reprod. Update 2016, 22, 306-319. [CrossRef] [PubMed]

3. Ferraretti, A.P.; La Marca, A.; Fauser, B.C.J.M.; Tarlatzis, B.; Nargund, G.; Gianaroli, L. ESHRE consensus on the definition of poor response to ovarian stimulation for in vitro fertilization: The Bologna criteria. Hum. Reprod. 2011, 26, 1616-1624. [CrossRef]

4. Vaiarelli, A.; Cimadomo, D.; Ubaldi, N.; Rienzi, L.; Ubaldi, F.M. What is new in the management of poor ovarian response in IVF? Curr. Opin. Obstet. Gynecol. 2018, 30, 155-162. [CrossRef]

5. Jeve, Y.B.; Bhandari, H.M. Effective treatment protocol for poor ovarian response: A systematic review and meta-analysis. J. Hum. Reprod. Sci. 2016, 9, 70-81. [CrossRef] [PubMed]

6. Williams, C.J.; Erickson, G.F. Morphology and Physiology of the Ovary; Feingold, K.R., Anawalt, B., Boyce, A., Chrousos, G., de Herder, W.W., Dungan, K., Grossman, A., Hershman, J.M., Hofland, H.J., Kaltsas, G., et al., Eds.; MDText.com, Inc.: South Dartmouth, MA, USA, 2000.

7. Knight, P.G.; Glister, C. TGF- $\beta$ superfamily members and ovarian follicle development. Reproduction 2006, 132, 191-206. [CrossRef] [PubMed]

8. Knobil, E.; Neill, J.D. The Physiology of Reproduction, 2nd ed.; Raven Press: New York, NY, USA, 1994.

9. Lee, J.H.; Cho, Y.I.; Choi, S.S.; Kim, H.-W.; Min, C.K.; Lee, S.J. Loss and gain-of-function approach to investigate early cell fate determinants in preimplantation mouse embryos. J. Vis. Exp. 2016, 112, 53696. [CrossRef] [PubMed]

10. Wilson, N.H.; Key, B. Neogenin: One receptor, many functions. Int. J. Biochem. Cell Biol. 2007, 39, 874-878. [CrossRef] [PubMed]

11. Dun, X.-P.; Parkinson, D.B. Role of Netrin-1 signaling in nerve regeneration. Int. J. Mol. Sci. 2017, 18, 491. [CrossRef]

12. Fujita, Y.; Yamashita, T. The roles of RGMa-neogenin signaling in inflammation and angiogenesis. Inflamm. Regen. 2017, 37, 6. [CrossRef]

13. Hagihara, M.; Endo, M.; Hata, K.; Higuchi, C.; Takaoka, K.; Yoshikawa, H.; Yamashita, T. Neogenin, a receptor for bone morphogenetic proteins. J. Biol. Chem. 2011, 286, 5157-5165. [CrossRef] [PubMed]

14. Kam, J.W.K.; Dumontier, E.; Baim, C.; Brignall, A.C.; Da Silva, D.M.; Cowan, M.; Kennedy, T.E.; Cloutier, J.-F. RGMB and neogenin control cell differentiation in the developing olfactory epithelium. Development 2016, 143, 1534-1546. [CrossRef] [PubMed]

15. Siebold, C.; Yamashita, T.; Monnier, P.P.; Mueller, B.K.; Pasterkamp, R.J. RGMs: Structural insights, molecular regulation, and downstream signaling. Trends Cell Biol. 2017, 27, 365-378. [CrossRef] [PubMed]

16. Rodriguez, A.; Pan, P.; Parkkila, S. Expression studies of neogenin and its ligand hemojuvelin in mouse tissues. J. Histochem. Cytochem. 2006, 55, 85-96. [CrossRef] [PubMed]

17. Lee, J.H.; Choi, S.S.; Kim, H.W.; Xiong, W.C.; Min, C.K.; Lee, S.J. Neogenin as a receptor for early cell fate determination in preimplantation mouse embryos. PLoS ONE 2014, 9, e101989. [CrossRef] [PubMed]

18. Sánchez, F.; Smitz, J. Molecular control of oogenesis. Biochim. Biophys. Acta Mol. Basis Dis. 2012, 1822, 1896-1912. [CrossRef]

19. Coutandin, D.; Osterburg, C.; Srivastav, R.K.; Sumyk, M.; Kehrloesser, S.; Gebel, J.; Tuppi, M.; Hannewald, J.; Schäfer, B.; Salah, E.; et al. Quality control in oocytes by 663 is based on a spring-loaded activation mechanism on the molecular and cellular level. eLife 2016, 5. [CrossRef]

20. Johnson, E.T.; Nicola, T.; Roarty, K.; Yoder, B.K.; Haycraft, C.J.; Serra, R. Role for primary cilia in the regulation of mouse ovarian function. Dev. Dyn. 2008, 237, 2053-2060. [CrossRef]

21. Mattson, B.A.; Albertini, D.F. Oogenesis: Chromatin and microtubule dynamics during meiotic prophase. Mol. Reprod. Dev. 1990, 25, 374-383. [CrossRef]

22. Carnegie, J.A.; Dardick, I.; Tsang, B.K. Microtubules and the gonadotropic regulation of granulosa cell steroidogenesis. Endocrinology 1987, 120, 819-828. [CrossRef]

23. Scantland, S.; Tessaro, I.; Macabelli, C.H.; Macaulay, A.D.; Cagnone, G.; Fournier, É.; Luciano, A.M.; Robert, C. The adenosine salvage pathway as an alternative to mitochondrial production of ATP in maturing mammalian oocytes. Biol. Reprod. 2014, 91, 75. [CrossRef]

24. Ervin, J.; Schütz, L.; Spicer, L. Current status of the role of endothelins in regulating ovarian follicular function: A review. Anim. Reprod. Sci. 2017, 186, 1-10. [CrossRef]

25. Ko, C. Endothelins in regulating ovarian and oviductal function. Front. Biosci. 2011, 3, 145-155. [CrossRef] [PubMed]

26. Cacioppo, J.A.; Oh, S.W.; Kim, H.-Y.; Cho, J.; Lin, P.-C.P.; Yanagisawa, M.; Ko, C. Loss of function of endothelin-2 leads to reduced ovulation and CL formation. PLoS ONE 2014, 9, e96115. [CrossRef] [PubMed]

27. Farhat, A.; Philibert, P.; Sultan, C.; Poulat, F.; Boizet-Bonhoure, B. Hematopoietic-prostaglandin D2 synthase through PGD2 production is involved in the adult ovarian physiology. J. Ovarian Res. 2011, 4, 3. [CrossRef]

28. Niringiyumukiza, J.D.; Cai, H.; Xiang, W. Prostaglandin E2 involvement in mammalian female fertility: Ovulation, fertilization, embryo development and early implantation. Reprod. Biol. Endocrinol. 2018, 16, 1-10. [CrossRef] [PubMed]

29. Copland, J.A.; Zlatnik, M.G.; Ives, K.L.; Soloff, M.S. Oxytocin receptor regulation and action in a human granulosa-lutein cell line. Biol. Reprod. 2002, 66, 1230-1236. [CrossRef] [PubMed]

30. Pierzynski, P.; Reinheimer, T.M.; Kuczynski, W. Oxytocin antagonists may improve infertility treatment. Fertil. Steril. 2007, 88, 213.e19-213.e22. [CrossRef] [PubMed] 
31. Jahromi, B.N.; Dabbaghmanesh, M.H.; Bakhshaie, P.; Parsanezhad, M.E.; Anvar, Z.; Alborzi, M.; Zarei, A.; Bakhshaei, M. Assessment of oxytocin level, glucose metabolism components and cutoff values for oxytocin and anti-mullerian hormone in infertile PCOS women. Taiwan J. Obstet. Gynecol. 2018, 57, 555-559. [CrossRef] [PubMed]

32. Evans, J.; Reid, R.; Wakeman, S.; Croft, L.; Benny, P. Evidence that oxytocin is a physiological component of LH regulation in non-pregnant women. Hum. Reprod. 2003, 18, 1428-1431. [CrossRef] [PubMed]

33. Assou, S.; Haouzi, D.; de Vos, J.; Hamamah, S. Human cumulus cells as biomarkers for embryo and pregnancy outcomes. Mol. Hum. Reprod. 2010, 16, 531-538. [CrossRef] [PubMed]

34. Feuerstein, P.; Cadoret, V.; Dalbies-Tran, R.; Guerif, F.; Bidault, R.; Royere, D. Gene expression in human cumulus cells: One approach to oocyte competence. Hum. Reprod. 2007, 22, 3069-3077. [CrossRef] [PubMed]

35. Huang, Z.; Wells, D. The human oocyte and cumulus cells relationship: New insights from the cumulus cell transcriptome. Mol. Hum. Reprod. 2010, 16, 715-725. [CrossRef] [PubMed]

36. Dumesic, D.A.; Meldrum, D.R.; Katz-Jaffe, M.G.; Krisher, R.L.; Schoolcraft, W.B. Oocyte environment: Follicular fluid and cumulus cells are critical for oocyte health. Fertil. Steril. 2015, 103, 303-316. [CrossRef] [PubMed] 